\title{
Spherical Coordinate-based Reliability Analysis in a Control System
}

\author{
Xuexuan ZHU \\ School of Mechanical Engineering Xinyu University, Jiangxi Xinyu 338004, China \\ 371734429@qq.com
}

\begin{abstract}
It is very important to design a feedback loop which takes the output into consideration so that the system is able to adjust its performance. Reliability analysis in the control system is able to examine the system stability and make the control system is more compatible and reliable. This paper introduces a spherical coordinate-based reliability analysis model to examine the feedback in a control system for improving the control signal between encoder and decoder of the controller. Under the feedback closed-loop system with erasing operations and limited coding ratio, the proposed spherical coordinated-based approach is able to ensure the average asymptotic reliability. To this end, the design methodology of controller, encoder, decoder, and quantifier are introduced. From the experiments analysis, it could be observed that the proposed approach is able to achieve high system reliability. It is observed that, under the bigger erasing possibility, the designed quantifier and controller are able to ensure the system average asymptotic reliability.
\end{abstract}

Keywords: Spherical Coordinate, Reliability Analysis, Control System, Information Channel

\section{Introduction}

When talking about the control system, it is very important to design a feedback loop which takes the output into consideration so that the system is able to adjust its performance or make some decisions to satisfy a desired response [1]. A basic feedback structure uses the output value of the system to assist the preparation of the next output. Such control mechanisms are widely used in existing systems such as actuators, sensors, motors, and other electronic devices through setting some parameters [2]. A closed loop system has a feedback control so that the reliability of the system could be enhanced when carrying out some decision-makings [3-4]. Considering the system reliability under the control channel communication with discrete linear signal, the noise will cause the error coding or signal in the whole control system. For example, the noise may locates on the channel between sensors and controllers. How to solve the problem when the noise intervene the normal control signal so that the accurate feedback or response could be implemented is very challenging.

Reliability analysis in the control system is able to examine the system stability and make the control system is more compatible and reliable. In the literature, reliability engineering is termed to achieve either a prescribed reliability for a higher reliability at lower costs or a planned system [5-6]. In practice, take an automotive control system for an example, it is very complex with respect to the interconnected components, devices, and sensors among different parts which are connected by various control system via wireless or wired cables or communication channels [7]. Currently, large number of research has been carried out to study the quantitative reliability analysis in the control area. Considering the whole system visibility and reliability, a model predictive control system for a hybrid battery-ultracapacitor power source is proposed [8]. In this paper, the 
controller allocates fast current changes to the ultracapacitor and the battery responds mainly to slow current changes that helps to increase the battery lifetime. In order to combine the control and communication performance, the coding and decoding approach is used for working out the design solution. [9] presents a funnel dynamic surface control combined with fuzzy echo state networks (FESNs) for the prescribed tracking performance of a strict feedback multi-input-multi-output (MIMO) nonlinear dynamic system. This paper proposes a new funnel variable, thus, the funnel virtual control forces the tracking error to fall within funnel boundary. From the research, it is found that the necessary condition of the reliability and feasibility of the system under noise channel is $C^{c a p} \geq \sum \lambda(A) \max \{0, \log |\lambda(A)|\}$. where, $\lambda(A)$ is the eigenvalue of the system matrix, $C^{c a p}$ is defined as $\liminf _{T \rightarrow \infty} \frac{1}{T} C_{T}^{c a p}, C_{T}^{c a p}$ is the Shannon capacity of the time $T$. In order to ensure the reliability of the matrix boundary, [10] introduced the concept of anytime capacity. This paper also presents the necessary and sufficient condition for the system matrix.

One of the critical components of the control system is encoder which uses the system status and control input from past and current. However, in practice, the encoder will cost a lot for achieving the purpose in terms of time and investment since it is very challenging to get the reliable and feasible minimum channel ratio. In order to handle this problem, spherical coordinate approach is used for enhancing the control signal over the channel so that the reliability of the feedback control system can be beneficial with the discrete linear system [11]. Spherical coordinate encoder uses the nonlinear transform from rectangular coordinate to spherical coordinate system, quantifying the spherical coordinate of the system status [12]. This paper introduces a spherical coordinate-based reliability analysis model to examine the feedback in a control system for improving the control signal between encoder and decoder of the controller.

The rest of this paper is structured as follows. Section 2 illustrates the problem description of the discrete linear control system and gives several definitions of the control system with noise channel. Under the definition, section 3 reports on the spherical coordinate-based approach to design the control system encoder, decoder, controller and quantifier. Section 4 talks about the experiments and discusses the results. Key observations are obtained in this section too. Conclusions are organized in section 5 which gives the research directions to improve this research.

\section{Problem Description}

Considering the following discrete linear system $x(k+1)=A x(k)+B u(k)$, where $x(k) \in \square^{d}$ and $u(k) \in \square^{m}$ are the system status and input. $A$ and $B$ are the $n$ dimensional matrix. Assume that $A$ is unstable and the initial status is $x(0)$, which is a boundary area that $\|x(0)\|_{2} \leq \bar{r} . \bar{r}$ is known for the encoder and decoder. The system structure is shown in Figure 1 which presents the control system feedback. 


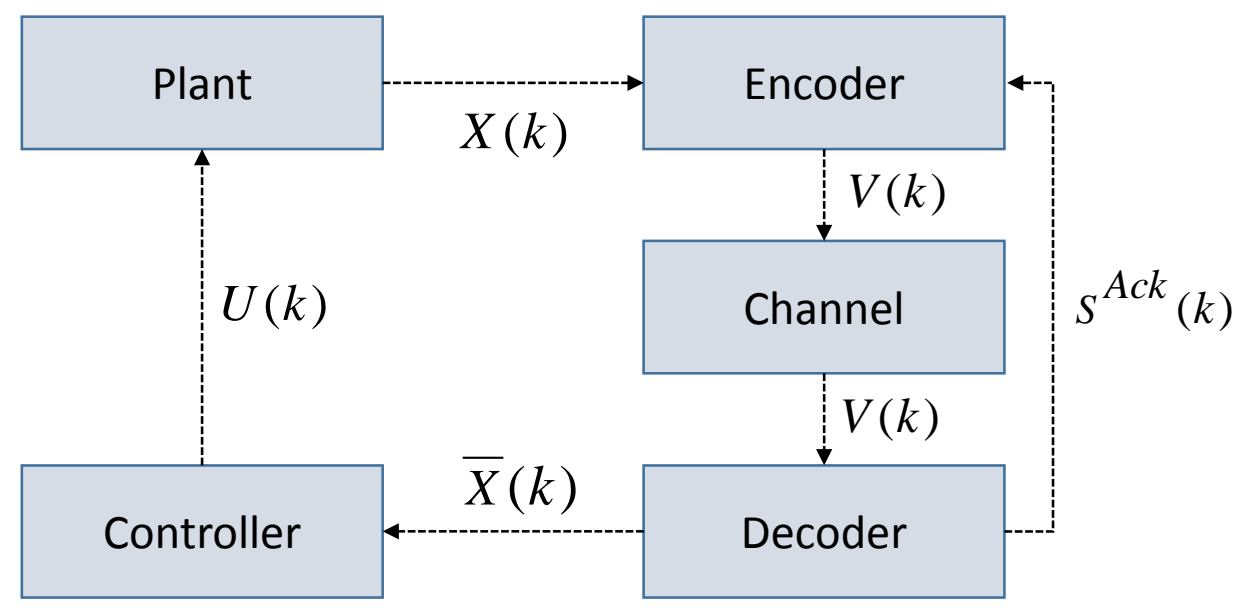

Figure 1. System Structure

From Figure 1, the encoder is able to encode the status $x(k)$. After that, it is sent to decoder via channel. The erasing probability in the channel is defined as $p$. The decoder will send back a confirm signal to the encoder so as to ensure whether the erase operation occurs or not. Thus, the encoder knows what message will receive [13]. The confirmation signal is $S^{A c k}(k) \in\{0,1\}$, which presents the signal is in time $k$. The signal is sent by the decoder and encoder will receive it. The random variable is defined as follows:

$S^{A c k}(k)=\left\{\begin{array}{l}0, N \\ 1, Y\end{array}\right.$

$S^{A c k}(k), k=0,1, \ldots, n$ is independent distribution which is:

$P_{r}\left(S^{A c k}(k)=0\right)=1-p, P_{r}\left(S^{A c k}(k)=1\right)=p$

$N$ is no erasing operation at time $k$ and $Y$ presents there is an erasing.

The control system follows the procedures:

(1) Encoder: at time $k$, the encoder is a mapping $\varepsilon_{k}$ from $\left(x(k), S^{A c k}(k-1)\right)$ to $V(k) \in \sum . V(k)=\varepsilon_{k}\left(x(k), S^{A c k}(k-1)\right)$

(2) Erasing channel: encoder send the $V(k)$ in channel sending point. Due to the noise, it will be $\overline{\bar{V}}(k)$ in the decoder. The possibility will be $P_{r}(\overline{\bar{V}}(k)=V(k))=1-p, P_{r}(\overline{\bar{V}}(k)=e)=p, e$ is the erasing operator which presents the erasing operation occurs.

(3) Decoder: the decoder at time $k$ is a mapping $D_{k}$ from $\left(\overline{\bar{V}}(k), S^{A c k}(k-1)\right)$ to $\bar{x}(k), \bar{x}(k)$ is the estimation of $x(k) . \bar{x}(k)=D_{k}\left(\overline{\bar{V}}(k), S^{A c k}(k-1)\right) \in \square d$.

(4) Controller: controller $\square$ is a mapping from $\bar{x}(k)$ to $u(k) \in \square^{m}$, $u(k)=\square(\bar{x}(k)) \in \square^{m}$. The status feedback control is $u(k)=K(\bar{x}(k))$ and the feedback incremental matrix is $K$. 
In order to define the reliability of the control system, there are several definitions:

Definition 1. A control system with noise channel is averagely stable. If there is an encoder, decoder, and controller form a closed-loop system, $0 \in \square^{d}$ is the balanced point, and $E\left(\left\|x_{k}\right\|_{2}\right) \rightarrow 0, k \rightarrow \infty$, where $E(\cdot)$ is the expectation.

Definition 2. A control system with noise channel is averagely stable. If there is an encoder, decoder, and controller form a closed-loop system, $0 \in \square^{d}$ is the balanced point, when $k \rightarrow \infty,\left\|x_{k}\right\|_{2} \rightarrow 0$.

\section{Spherical Coordinate-based Encoding Approach}

\subsection{Encoding}

At time $k$, according to the confirmed signal $S^{A c k}(k-1)$ and system behavior, encoder $\varepsilon_{k}$ updater's parameter $L_{k}$ of $\left(L_{k}, N, a, M\right)$, which is the supporting ball $\Lambda_{k}$ whose quantified block number of $2(N-1) M^{d-1}+1 . x(k)$ is encoded into $V(k)$. That means the number of quantified block $x(k)$ is $V(k)$.

\subsection{Decoding}

If the channel has no erasing operation, $\overline{\bar{V}}(k)=V(k)$ exists. Decoder sends feedback the confirmation signal $S^{A c k}(k)=0$ to the encoder. Meanwhile, if $\overline{\bar{V}}(k)$ presents the quantified blocks indexed by $\left(i, i_{1}, \ldots, i_{d-2}, s\right)$. Decoder converts the $\bar{x}(k)$ into spherical coordinate:

$$
\begin{aligned}
& r=\frac{(1+a)}{(1+2 a)^{N-1-i}} L, \theta_{n}=\left(i_{n}+\frac{1}{2}\right) \frac{\pi}{M}, n=1,2, \ldots, d-2 \\
& \theta_{d-1}=\left(s+\frac{1}{2}\right) \frac{\pi}{M}
\end{aligned}
$$

If $\overline{\bar{V}}(k)$ presents $S_{1}(k)$, let $\bar{x}(k)=0$. If the channel has erasing operations, $\overline{\bar{V}}(k)=e$. The confirmation signal $S^{A c k}(k)=1$ will be fed back to encoder and $\bar{x}(k)=0$. In this case, if $\overline{\bar{V}}(k)$ presents the quantified block $S_{1}(k)$ or there some erasing operations occur, $\bar{x}(k)=0$ always exist. Then the balanced point is $0 \in Z^{d}$.

\subsection{System Controller Design}

Based on the spherical coordinate encoding approach, the design of the status feedback is $u(k)=K^{-}(k)$. The status estimation of the controller is $\bar{x}(k)=x(k)+e_{x}(k)$, where $e_{x}(k)$ is the quantified error [14]. Thus, the closed-loop feedback system is:

$$
x(k+1)=A x(k)+B K^{-}(k)=(A+B K) x(k)+B K e_{x}(k)
$$


Lemma 1: Define $\Lambda_{k+1}=\left\{\begin{array}{c}\left\{x \in \square:\|x\|_{2} \leq \mu L_{k}\right\}, \text { if } S^{A c k}(k)=0 \\ \left.\{x \in]^{d}:\|x\|_{2} \leq \delta(A) L_{k}\right\}, \text { if } S^{A c k}(k)=1\end{array} \quad\right.$,if $x(k) \in \Lambda_{k}$, then $x(k+1) \in \Lambda_{k+1} . \quad \mu=\max \left\{\delta(A+B K)+\eta \delta(B K), \frac{\delta(A)}{(1+2 a)^{N-1}}\right\}, \quad \eta=\left\{a+(d-1) \frac{\pi}{2 m}\right\} . \quad \delta(\cdot)$ presents the maximum singular value of the matrix.

Proof. Let $S^{A c k}(k)=0$, if $x(k) \in S_{N}(k) \backslash S_{1}(k)$, then we can get $\|x(k+1)\|_{2}=\left\|(A+B K) x(k)+B K e_{x}(k)\right\|_{2} \leq(\delta(A+B K)+\eta \delta(B K))\|x(k)\|_{2} \leq(\delta(A+B K)+\eta \delta(B K)) L_{k}$. If $x(k) \in S_{1}(k)$, then $\bar{x}(k)=0$ and $\|x(k+1)\|_{2}=\|\left(A x(k) \|_{2} \leq\left(\delta(A) \|\left(x(k) \|_{2} \leq \frac{\delta(A) L_{k}}{(1+2 a)^{N-1}}\right.\right.\right.$. Then $\|x\|_{2} \leq \mu L_{k}$.

Let $S^{A c k}(k)=1$, in this case, $\quad \bar{x}(k)=0$ and $x(k) \in \Lambda_{k}$, thus, $\|x(k+1)\|_{2} \leq \delta(A) \|\left(x(k) \|_{2} \leq \delta(A) L_{k}\right.$.

Based on the lemma and according to the equation $L_{k+1}=\left\{\begin{array}{c}\mu L_{k}, \text { if } S^{A c k}(k)=0 \\ \delta(A) L_{k}, \text { if } S^{A c k}(k)=1\end{array}\right.$, the parameter $L_{k+1}$ could be updated by the quantified controller $\left(L_{k+1}, N, a, M\right)$.

Based on the theoretical analysis, the design of the control system is as follows:

Design of controller. In order to find the value of $K$ and $P . P$ is a reversible matrix and $\bar{x}(k)=P x(k)$. s.t. $\delta\left(P(A+B K) P^{-1}\right)(1-p)+\delta\left(P A P^{-1}\right) p<1$.

Design of quantifier. After obtaining the $K$ and $P, a$ and $M$ could be selected to meet $\left[\delta\left(P(A+B K) P^{-1}\right)+\eta \delta\left(P(B K) P^{-1}\right)\right]^{(1-p)}\left[\delta\left(P A P^{-1}\right)\right]<1$, select $N$ s.t.

$\frac{\delta\left(P A P^{-1}\right)}{(1+2 a)^{N-1}} \leq \delta\left(P(A+B K) P^{-1}\right)+\eta \delta\left(P(B K) P^{-1}\right)$

We can get $L_{0}=\delta(\overline{P r})$.

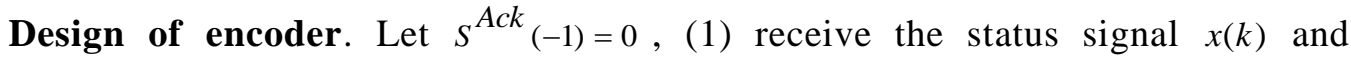
convert it to $\bar{x}(k)=P x(k)$; (2) use the quantifier $\left(L_{k}, N, a, M\right)$ to convert the $\bar{x}(k)$ coding to $V(k)=\varepsilon_{k}\left(\bar{x}(k), S^{A c k}(k-1)\right)$; (3) send the $V(k)$ to decoder and receive the confirmation signal $S^{A c k}(k)$; (4) update the time $k=k+1$ and return to step (1).

Design of decoder. The design follows four steps: (1) at time $k$, if there is no erasing operation, assume $S^{A c k}(k)=0, \overline{\bar{V}}(k)=V(k)$. If there are several erasing operations, $\quad S^{A c k}(k)=1$ and $\overline{\bar{V}}(k)=e ; \quad(2) \quad$ decode the $\overline{\bar{V}}(k)$ to $\overline{\bar{V}}^{\prime}(k)=D_{k}\left(\overline{\bar{V}}(k), S^{A c k}(k-1)\right)$, send the confirmation signal $S^{A c k}(k)$ to the encoder; (3) convert the $\bar{V}^{\prime}(k)$ into $\bar{x}(k)=P^{-1} \overline{\bar{V}}^{\prime}(k)$; (4) update the time $k=k+1$ and return to step (1). 
In order to ensure the quantifier working mechanism, the ratio of channel $R$ should satisfy $R \geq\left\lceil\log _{2}\left(2(N-1) M^{d-1}+1\right\rceil .\lceil\cdot\rceil\right.$ is the ceiling function.

\subsection{Reliability Analysis}

First of all, a suitable reliability index $0<\gamma<1$ is selected. The following matrix inequality of matrix $Q$ and $W$ should be worked out to examine the reliability.

$$
\begin{aligned}
& \left(\begin{array}{cc}
-\left(\frac{1-\gamma}{p}\right)^{2} Q & A Q \\
Q^{T} A^{T} & -Q
\end{array}\right)<0 \\
& \left(\begin{array}{cc}
-\left(\frac{1-\gamma}{p}\right)^{2} Q & A Q+B W \\
Q^{T} A^{T}+W^{T} B^{T} & -Q
\end{array}\right)<0
\end{aligned}
$$

We can get $K=W Q^{-1}$. Based on the Cholesky decomposition, $P$ could be calculated by $Q=P^{-1} P^{-T}$. Using $K=W Q^{-1}$ and $Q=P^{-1} P^{-T}$, inequality (7)(8) could be:

$$
\begin{aligned}
& \left(\begin{array}{cc}
-\left(\frac{1-\gamma}{p}\right)^{2} P^{-1} P^{-T} & A P^{-1} P^{-T} \\
P^{-1} P^{-T} A^{T} & -P^{-1} P^{-T}
\end{array}\right)<0 \\
& \left(\begin{array}{cc}
-\left(\frac{1-\gamma}{p}\right)^{2} P^{-1} P^{-T} & (A+B K) P^{-1} P^{-T} \\
P^{-1} P^{-T}(A+B K)^{T} & -P^{-1} P^{-T}
\end{array}\right)<0
\end{aligned}
$$

$\operatorname{diag}[P, I]$ is used to left and right multiplication [15] of (9) and (10), then we can get:

$$
\begin{aligned}
& \left(\begin{array}{cc}
-\left(\frac{1-\gamma}{p}\right)^{2} I & P A P^{-1} P^{-T} \\
P^{-1} P^{-T} A^{T} P^{T} & -P^{-1} P^{-T}
\end{array}\right)<0 \\
& \left(\begin{array}{cc}
-\left(\frac{1-\gamma}{p}\right)^{2} I & P(A+B K) P^{-1} P^{-T} \\
P^{-1} P^{-T}(A+B K)^{T} P^{T} & -P^{-1} P^{-T}
\end{array}\right)<0
\end{aligned}
$$

Using Schur Lemma, we can get:

$$
\begin{aligned}
& P A P^{-1} P^{-T} A^{T} P^{T}<\left(\frac{1-\gamma}{p}\right)^{2} I \\
& P(A+B K) P^{-1} P^{-T}(A+B K)^{T} P^{T}<\left(\frac{1-\gamma}{p}\right)^{2} I
\end{aligned}
$$

Then the reliability of the control system could be examined by the following two indicators: 
$\delta\left(P A P^{-1}\right) p<1-\gamma$

$\delta\left(P(A+B K) P^{-1}\right)(1-p)<\gamma$

\section{Experiments and Results Analysis}

The experiments are carried out to verify the feasibility of the proposed spherical coordinate-based reliability analysis of a control system. The experiments matrix is as follows:

$$
A=\left[\begin{array}{cccc}
1.2 & 0 & 0 & 0 \\
0 & -1 & 0 & 0 \\
0 & 0 & -1.5 & -1.5 \\
0 & 0 & 0 & -1
\end{array}\right], B=\left[\begin{array}{cc}
1 & 1 \\
-0.2 & 0.2 \\
0.2 & 1 \\
1 & 0.2
\end{array}\right]
$$

Matrix A has the characteristic value $[1.2,-1,-1.5,-1]$, and $(A, B)$ is controllable. Let the erasing possibility $p=0.2$. For the average asymptotic reliability of the system, we can get:

$$
Q=\left[\begin{array}{cccc}
28.68 & -6.25 & -19.38 & -6.00 \\
-6.25 & 3.72 & 9.16 & 2.19 \\
-19.38 & 9.16 & 25.51 & 7.18 \\
-6.00 & 2.19 & 7.18 & 2.45
\end{array}\right] \text { and } W^{T}=\left[\begin{array}{cc}
-2.31 & -27.05 \\
-0.04 & 9.39 \\
1.64 & 26.22 \\
1.22 & 7.11
\end{array}\right]
$$

Then $K=W Q^{-1}=\left[\begin{array}{cccc}-0.02 & 1.09 & -1.13 & 2.77 \\ -0.53 & -2.32 & 2.44 & -3.46\end{array}\right]$. Using the Cholesky decomposition, we can get:

$$
P=\left[\begin{array}{cccc}
0.28 & -0.88 & 0.65 & -0.45 \\
0 & 3.81 & -2.36 & 3.50 \\
0 & 0 & 0.48 & -1.40 \\
0 & 0 & 0 & 0.64
\end{array}\right] \text { which meets } Q=P^{-1} P^{-T}
$$

For the quantifier parameters $\left(L_{k}, N, a, M\right): \eta=0.03, a=0.002, M=180, N=436$. $\eta=\left\{a+(d-1) \frac{\pi}{2 m}\right\}$. Then we can get $R \geq 33$. Figure 1 shows the experiments results of the response of the system status $x$ under average asymptotic stability with $p=0.2$ and indication of the region which the status $\bar{x}(k)$ of the control system lies in with $p=0.2$.

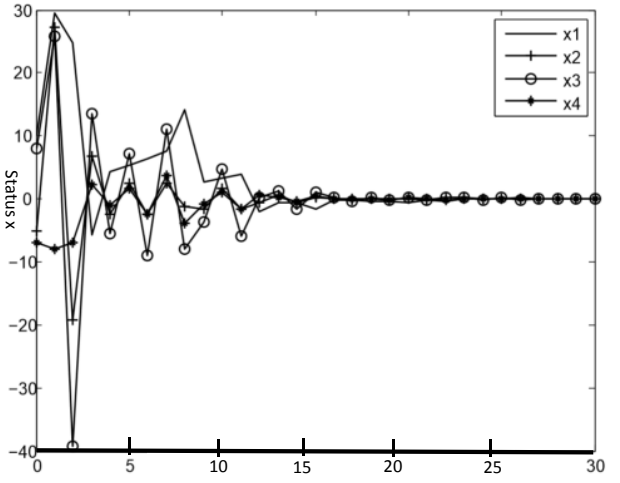

(a) Status $x$ under average asymptotic reliability

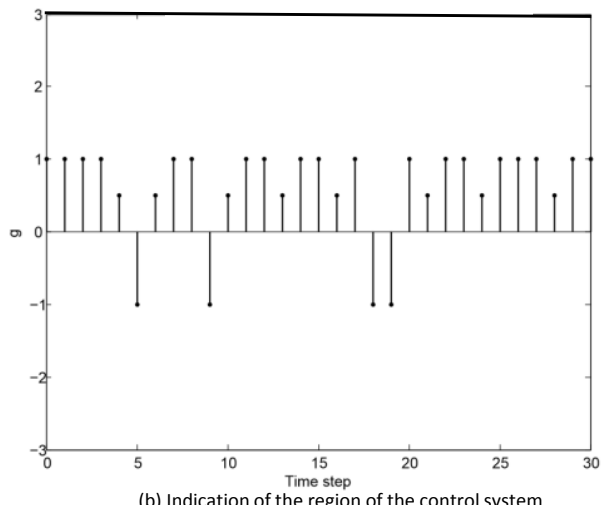

(b) Indication of the region of the control system

Figure 1. Experimental Results 
From Figure 1, the status curve shows the results. It is observed that the initial status $x(0)=[10,-5,8,-7]^{T}$ and the boundary $\bar{b}=16$. Then, $g(k)$ presenting which areas of supporting spherical will be located of $\bar{x}(k)$ as shown in Figure $1(b) . g(k)$ is defined as:

$$
g(k):=\left\{\begin{array}{c}
1, i f \frac{L_{K}}{(1+2 a)^{N-1}}<\|\bar{x}(k)\|_{2} \leq L_{k} \\
0.5, i f\|\bar{x}(k)\|_{2} \leq \frac{L_{K}}{(1+2 a)^{N-1}} \\
-1, \text { others }
\end{array}\right.
$$

Using the same quantifier and controller from Figure 1 (b), when the erasing possibility $p=0.1$, the status curve and function $g(k)$ is almost the same. It is observed that, under the bigger erasing possibility, the designed quantifier and controller are able to ensure the system average asymptotic reliability.

Similarly, another experiment is carried out to compare with the previous experimental scenario. We can get:

$$
\begin{gathered}
K=\left[\begin{array}{cccc}
-0.06 & 1.80 & -1.56 & 3.32 \\
-0.52 & -1.28 & 1.85 & -2.64
\end{array}\right] \\
P=\left[\begin{array}{cccc}
3.59 & 3.64 & 0.75 & 4.26 \\
0 & 9.12 & -5.26 & 7.57 \\
0 & 0 & 2.05 & -0.26 \\
0 & 0 & 0 & 1.85
\end{array}\right]
\end{gathered}
$$

With the quantifier parameters : $\left(L_{k}, N, a, M\right): \eta=0.06, a=0.007, M=87, N=152$. Then we can get $R \geq 28$. Comparing with the previous experiment, the coding ratio is not changed significant. It is found that the designed control system has a stable reliability based on the spherical coordinate-based approach.

\section{Conclusion}

This paper discusses the control system reliability considering the erasing operations in the channel. Under the feedback closed-loop system with erasing operations and limited coding ratio, the proposed spherical coordinated-based approach is able to ensure the average asymptotic reliability. To this end, the design methodology of controller, encoder, decoder, and quantifier are introduced. From the experiments analysis, it could be observed that the proposed approach is able to achieve high system reliability. Additionally, the bigger erasing possibility has little impacts on the system reliability because the confirmation signal is verified by the encoder and decoder by designed mechanisms.

Future research will be carried out from several perspectives. Firstly, this paper did not consider the situation that the encoder cannot get the control input. It is very challenging to get the minimum channel coding ratio under this situation. How to address this problem need further investigation. Secondly, the minimum coding ratio is $R_{\text {min }}=\frac{p}{1-p} \sum_{\lambda(A)} \max (0, \log |\lambda(A)|\}$. However, in order to ensure the variety of quantifier and controller with system asymptotic reliability, this indicator could be used for modelling the channel coding ratio so as to reduce the error ratio of a control system. Finally, the experiments carried out in this paper is based on two scenarios. 
In the future, more considerations could be implemented for example, the random variable $\Delta(k)$ is independent distribution maybe extended into the model.

\section{References}

[1] T. Atalik, M. Deniz, E. Koç, C. Ö. Gerçek, B. Gultekin and M. Ermis, "Multi-DSP and-FPGA-based fully digital control system for cascaded multilevel converters used in FACTS applications", Industrial Informatics, IEEE Transactions on, vol. 8, (2012), pp. 511-527.

[2] B. Talha and M. Patzold, "Channel models for mobile-to-mobile cooperative communication systems: A state of the art review", Vehicular Technology Magazine, IEEE, vol. 6, (2011), pp. 33-43.

[3] R. Y. Zhong, Z. Li, A. L. Y. Pang, Y. Pan, T. Qu and G. Q. Huang, "RFID-enabled Real-time Advanced Planning and Scheduling Shell for Production Decision-making", International Journal of Computer Integrated Manufacturing, vol. 26, (2013), pp.649-662.

[4] R. Y. Zhong, Q. Y. Dai, T. Qu, G. J. Hu and G. Q. Huang, "RFID-enabled Real-time Manufacturing Execution System for Mass-customization Production", Robotics and Computer-Integrated Manufacturing, vol. 29, (2013), pp.283-292.

[5] M. Glukhikh, M. Moiseev and H. Richter, "An Approach for the Reliability Analysis of Automotive Control Systems", Proceeding of the Fourth International Conference on Dependability, (2011), pp. 5156 ,.

[6] R. Y. Zhong, Q. Y. Dai, K. Zhou and X. B. Dai, "Design and Implementation of DMES Based on RFID", Proceeding of the $2^{\text {nd }}$ International Conference on Anti-counterfeiting, Security and Identification, Guiyang, pp. 475-477, (2008) August 20-23.

[7] Q. Y. Dai, R. Y. Zhong, G. Q. Huang, T. Qu, T. Zhang and T. Y. Luo, "Radio frequency identificationenabled real-time manufacturing execution system: a case study in an automotive part manufacturer", International Journal of Computer Integrated Manufacturing, vol. 25, (2012), pp. 51-65.

[8] B. Hredzak, V. G. Agelidis and M. Jang, "A model predictive control system for a hybrid batteryultracapacitor power source", IEEE Transactions on Power Electronics, vol. 29, (2014), pp. 1469-1479.

[9] S. I. Han and J. M. Lee, "Fuzzy echo state neural networks and funnel dynamic surface control for prescribed performance of a nonlinear dynamic system, IEEE Transactions on Industrial Electronics, vol. 61, (2014), pp. 1099-1112.

[10] P. García, C. A. Garcia, L. M. Fernández, F. Llorens and F. Jurado, "ANFIS-based control of a gridconnected hybrid system integrating renewable energies, hydrogen and batteries", IEEE Transactions on Industrial Informatics, vol. 10, (2014), pp.1107-1117.

[11] K. Liu, H. Skibbe, T. Schmidt, T. Blein, K. Palme and T. Brox, "Rotation-invariant HOG descriptors using fourier analysis in polar and spherical coordinates," International Journal of Computer Vision, vol. 106, (2014), pp.342-364.

[12] F. Mitri, "Partial-wave series expansions in spherical coordinates for the acoustic field of vortex beams generated from a finite circular aperture", IEEE Transactions on Ultrasonics, Ferroelectrics, and Frequency Control, vol. 61, (2014),pp. 2089-2097.

[13] R. Y. Zhong, G. Q. Huang, Q. Dai and T. Zhang, "Mining SOTs and dispatching rules from RFIDenabled real-time shopfloor production data", Journal of Intelligent Manufacturing, vol. 25, (2014), pp. 825-843.

[14] R. Y. Zhong, G. Q. Huang, S. L. Lan, Q. Y. Dai, T. Zhang and C. Xu, "A two-level advanced production planning and scheduling model for RFID-enabled ubiquitous manufacturing", Advanced Engineering Informatics, http://dx.doi.org/10.1016/j.aei.2015, vol. 01, no. 002, (2015).

[15] R. Y. Zhong, G. Q. Huang, S. Lan, Q. Dai, X. Chen and T. Zhang, "A big data approach for logistics trajectory discovery from RFID-enabled production data, International Journal of Production Economics, vol. 165, (2015), pp. 260-272.

\section{Authors}

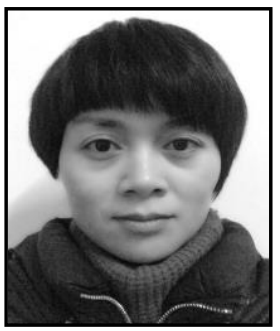

Xuexuan ZHU, Miss Zhu Xuexuan is a lecturer in School of Mechanical Engineering Xinyu University, Jiangxi Xinyu. She graduated from Nanchang University with bachelor of engineering and master of philosophy in 2001 and 2006 respectively. She has published about 10 papers in international journals and conferences. Her research interests include automation and control, algorithms and analysis, as well as electrical applications. 
International Journal of Signal Processing, Image Processing and Pattern Recognition Vol. 9, No. 5 (2016) 